\title{
Neoadjuvant Radio(chemo)therapy for Breast Cancer: An Old Concept Revisited
}

\author{
Christiane Matuschek $^{a} \quad$ Carolin Nestle-Kraemling $^{b}$ Thorsten Kühn ${ }^{c}$ \\ Tanja Fehm ${ }^{d}$ Edwin Bölke ${ }^{a}$ Stefanie Corradini ${ }^{e} \quad$ Gerd Fastner $^{f}$ Kitti Maas $^{a}$ \\ Clemens Seidel $^{g}$ Wilfried Budach ${ }^{a}$

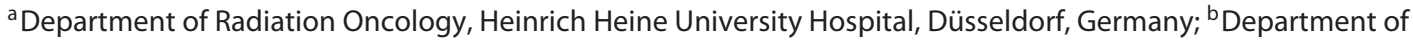 \\ Gynecologic and Obstetrics, EVK Dusseldorf, Heinrich Heine University, Düsseldorf, Germany; ${ }^{\mathrm{c}}$ Department of

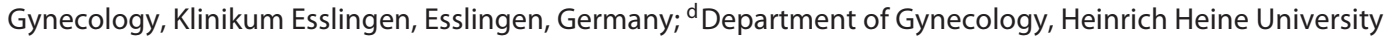 \\ Hospital, Düsseldorf, Germany; ${ }^{e}$ Department of Radiation Oncology, University Hospital, LMU Munich, Munich, \\ Germany; ${ }^{\mathrm{f} D e p a r t m e n t}$ of Radiotherapy and Radio-Oncology, Paracelsus Medical University, University Hospital \\ Salzburg, Landeskrankenhaus, Salzburg, Austria; ${ }^{9}$ Department of Radiation Oncology, University Hospital Leipzig, \\ Leipzig, Germany
}

\section{Keywords \\ Preoperative radiotherapy · Breast cancer · Neoadjuvant chemotherapy $\cdot$ Immediate reconstruction}

\section{Abstract}

Background: The international standard of care for the treatment of high-risk breast cancer (BC) consists of neoadjuvant chemotherapy (NACT) and surgery followed by adjuvant whole breast/chest wall irradiation. In this setting, the time interval from the start of NACT to the end of radiotherapy (RT) is usually postponed to 6 months or longer. In addition to this, a high percentage of capsular fibrosis may occur when breast implants are irradiated. Most of these disadvantages could be avoided by using preoperative RT (PRT). PRT is already the standard of care in several other tumor entities (rectal cancer, esophagus carcinoma, lung cancer, and soft tissue sarcoma). Nevertheless, PRT in BC has been tested in several trials, but randomized prospective trials using modern radiation technology and systemic therapies are lacking. The available evidence summarized in this review indicates that PRT may improve survival and reduce long-term toxicity in patients with a higher risk of recurrence and should be consequently tested in a randomized trial. Summary: Pro- spective, randomized trials concerning PRT in high-risk BC are needed. We plan to conduct a NeoRad trial (NACT followed by PRT in high-risk BC). Key Messages: Prospective, randomized studies concerning $\mathrm{PRT}$ in high-risk $\mathrm{BC}$ are needed.

(c) 2020 The Author(s)

Published by S. Karger AG, Basel

\section{Introduction}

The standard of care for the treatment of high-risk breast cancer (BC) consists of neoadjuvant chemotherapy (NACT) and surgery, followed by postoperative whole breast/chest wall irradiation with an optional tumor bed boost in the case of breast-conserving therapy. BC radiotherapy (RT) significantly reduces ipsilateral breast recurrences and BC-specific mortality. In addition, NACT achieves higher rates of pathological complete response ( $\mathrm{pCR})$, resulting in increased rates of breast-conserving surgery (BCS) or more accurate predictions of the chemosensitivity of BC [1]. If NACT were combined with preoperative RT (PRT), this approach could result in even higher rates of $\mathrm{pCR}$ and $\mathrm{BCS}$.

\section{The Author(s) \\ Karger} Published by S. Karger AG, Basel

Open access

This is an Open Access article licensed under the Creative Common Attribution-NonCommercial-40 International License (CC BY-NC) (http://www.karger.com/Services/OpenAccessLicense), applicable to the $/ /$ www.karger.com/Services/OpenAccessticense), applicable to
PD Dr. med. Christiane Matuschek

Department of Radiation Oncology, Heinrich Heine University Hospital Moorenstrasse 5

DE-40225 Düsseldorf (Germany)

Matuschek@med.uni-duesseldorf.de 
The concept of PRT has been well established for decades in several other tumor entities (e.g., rectal cancer, sarcoma, esophagus carcinoma, and lung cancer). Several publications regarding PRT in BC are available, but randomized prospective trials using modern radiation technology and systemic therapies are lacking [2]. We would like to conduct a prospective, international, multicenter, randomized phase 3 trial addressing this matter, i.e., NACT followed by PRT in high-risk BC (short title: NeoRad).

\section{NeoRad Trial}

The NeoRad trial tests whether PRT results in improved disease-free survival (DFS) and fewer radiationinduced late effects compared to postoperative RT in high-risk BC after NACT. The study design is shown in Figure 1. The aim of postoperative RT is to treat the residual locoregional microscopic disease in nonresected tissues. The overall treatment time for this residual microscopic disease from the first cycle of NACT to the completion of RT is about 3-6 weeks shorter, if RT is administered preoperatively. This should result in improved locoregional control, which is of special interest in view of the higher locoregional recurrence rates that have been reported after NACT compared to adjuvant chemotherapy [3]. Furthermore, residual disease potentially resistant to NACT has less time for metastatic spread.

While neoadjuvant RT is widely used in other tumor entities, there is still controversy regarding the risk of morbidity in breast surgery. Especially for BC, there is an ongoing debate about whether the application of neoadjuvant radiochemotherapy is safe. Even though the concept of PRT has been widely followed for the last 40 years, a concern is the risk of poor cosmetic results and impaired wound healing. This is presumably a misinterpretation of the term "preoperative" in delayed reconstruction or surgery of a recurrence tumor years later.

The advantages of PRT have been shown in randomized trials in other tumor entities, like rectal cancer and soft tissue sarcoma. In rectal cancer, neoadjuvant chemoradiation resulted in a significantly better local control and functional outcomes compared to postoperative chemoradiation [4]. Similarly, PRT of 50 Gy in 25 fractions in soft tissue sarcoma has been shown to be equivalent in terms of local tumor control to a $32 \%$ higher dose (66 Gy in 33 fractions) of postoperative RT. Late radiation induced tissue fibrosis was less pronounced after PRT and overall survival (OS) was significantly improved [5]. A major advantage of PRT concerning side effects is the lower dose required. In stage 3 non-small cell lung cancer, preoperative chemoradiation (45 Gy, 1.5 Gy BID in 3 weeks) was equivalent to postoperative chemoradiation
(54 Gy, 1.8 Gy in 6 weeks) in the intent-to-treat population. However, DFS and OS were better in the preoperative arm in patients who underwent surgical resection [6].

Regarding BC, a Scandinavian trial on stage 1-3 BC randomized 960 patients between 1971 and 1976 to receiving either mastectomy alone or mastectomy in combination with either PRT (45 Gy in 30 fractions) or postoperative RT. No additional systemic treatment was applied in that trial. In the first report of that trial a statistically significant advantage in OS $(\sim 7 \%)$ was observed at the 5-year followup in favor of the preoperative arm compared to both other treatment arms [7]. However, this survival advantage gradually disappeared during longer follow-up and it was no longer detectable at the 10-year follow-up [8]). Unfortunately, the causes of deaths were not well documented in this historic trial. Interestingly, a higher rate of mortality from any cause was observed in the preoperative arm of the trial compared to the postoperative arm, starting 4-5 years after treatment. This fact is unlikely to be caused by a higher BC mortality. In fact, the radiation techniques used were substantially different between the pre- and postoperative arms in that trial. Whereas in the preoperative arm photons were used to irradiate the breast and the internal mammary chain lymph nodes, electrons were used to irradiate the chest wall and the internal mammary chain lymph nodes in the postoperative arm. With the available radiation technique of the 1970s, this could have resulted in a large difference in the dose exposure of the heart in favor of the postoperative arm. The magnitude and time of occurrence of higher mortality rates in the preoperative arm fit well with the documented higher cardiovascular mortality associated with the type of RT used in the preoperative arm of the trial in a large meta-analysis of the Early Breast Cancer Trialists Cooperative Group [9]. Nowadays, even RT of left-sided BC including the internal mammary chain lymph nodes is no longer associated with such a significantly increased risk for cardiovascular mortality, which indirectly indicates that with the use of modern radiation techniques the survival benefit could have persisted after 5 years.

In a retrospective SEER database analysis the outcomes of 1,123 BC patients receiving PRT after NACT before surgery were compared to those of $155,077 \mathrm{pa}-$ tients who received surgery followed by postoperative RT [10]. The authors reported a $12 \%$ absolute benefit in DFS at 20 years for the preoperatively irradiated patients. The corresponding OS benefit was only $3 \%$, which could be explained by the fact that the majority of the patients in the database were treated in the last century starting from 1972. This indicates that the same problem applies as described above. Brackstone et al. [11] published a matched pair analysis that compared a small cohort of high-risk BC patients $(n=108)$ receiving PRT after NACT to postoperative RT. In this cohort, modern chemotherapy reg- 


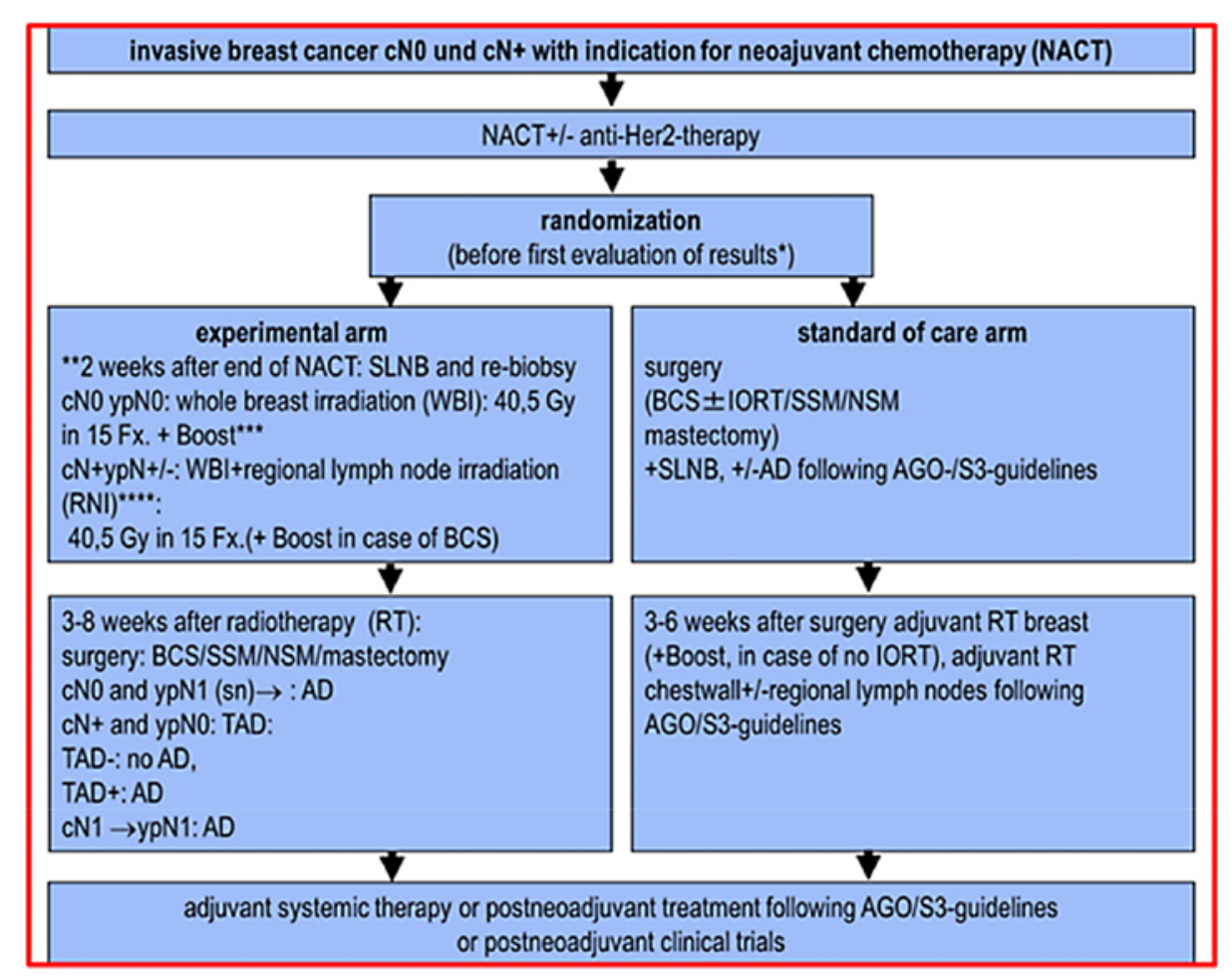

Abbreviations:

$\mathrm{AD}=$ Axillary dissection, $\mathrm{AGO}=$ Working group gynecological oncology, $\mathrm{BCS}=$ breast conserving surgery, $\mathrm{Fx}=$ Fractions, IORT= intraoperative radiotherapy, $\mathrm{NSM}=$ nipple sparing mastectomy, $\mathrm{n}=$ nodal, $\mathrm{SLNB}=$ sentinel node biopsy, $\mathrm{SSM}=$ skin sparing mastectomy, TAD = targeted axillary dissection

Fig. 1. Study design of the NeoRad trial.

Legend:
$\star$ Response evaluation using ultrasound following guidelines, optional MRI
before CHX and for re-evaluation following institutional procedure
$\star \star \star S L N B$ and second biopsy of the primary tumour before beginning of RT in
experimental arm to differentiate between effects of CHX and RT
$\star \star \star$ Sequential boost with $4 \times 2,7$ Gy per fraction up to 51,3 Gy or IORT Boost with
$1 \times 10$ Gy electrons or 20 Gy 50 kV-photons
$\star \star \star \star$ in case of left sided breast cancer, interruption of anti-Her2-therapy for 3
weeks (in dual blockade as well).

imens and modern radiation techniques were used. At 4 years an absolute advantage of $19 \%$ was observed for DFS and one of $14 \%$ was seen for OS, in favor of the preoperatively irradiated cohort. One may argue that the observation of a pCR after PRT and NACT cannot have the same predictive value as a $\mathrm{pCR}$ after NACT alone, since a higher pCR rate is expected after additional RT. This is potentially dangerous because some patients who are candidates for post-NACT or T-DM1 therapy will not receive this treatment. However, long-term survival data (15 years) from a larger cohort of 315 patients treated with preoperative chemotherapy and $\mathrm{RT}$ at the University Hospital Düsseldorf [12] clearly indicate that pCR rates after PRT and chemotherapy have the same impact on survival (no pCR, 55\% at 15 years; pCR, $85 \%$ at 15 years) as typically observed in trials using NACT alone [13-15].
Furthermore, retrospective data have shown that higher pCR rates after additional RT and NACT compared to sole NACT can be expected especially in luminal B BC, whereas a further increase in the already high $\mathrm{pCR}$ rates in Her2-positive and triple-negative $\mathrm{BC}$ will be less pronounced [2]. In order to minimize a potentially lower rate of patients receiving post-NACT or T-DM1 treatment when using PRT, an axillary sentinel node biopsy and a biopsy of the residual breast lesion are recommended for all patients (mandatory in Her2-positive and triple-negative cases) prior to PRT. For patients with triple-negative or Her2-positive disease, who have residual invasive cancer in either of these biopsies, postneoadjuvant treatment is even recommended in the case of a pCR after PRT.

In the era of modern RT techniques and systemic treatments, all of these considerations are the basis for the 
suggested randomized trial. Nowadays, several techniques such as intensity-modulated radiation therapy (IMRT), volumetric modulated arc therapy (VMAT) and respiratory gating techniques are available. IMRT is an advanced form of high-precision RT that allows delivery of the radiation beam in multiple small volumes with determined dose intensity patterns. The multiple intensitymodulated fields coming from different beam directions conform the shape of the tumor and minimize the dose to adjacent normal tissues. This technique is often used in irregular target volumes, e.g., lymph node irradiation in advanced BC including the mammaria interna lymph nodes. VMAT is a form of IMRT technique. In VMAT the RT machine rotates around the patient during an RT beam. This technique is also called "rapid arc." Respiratory gating monitors the breathing with a device and adjusts the radiation beam as necessary during treatment. The breath hold technique during irradiation of the left breast results in a significantly lower heart dose.

Summarized, the use of modern RT techniques can reduce the radiation exposure of at-risk organs, such as the lungs or the heart. This reduces the acute and late side effects of radiation therapy.

In the NeoRad trial the use of modern techniques is standard and will be part of the study protocol.

In addition to advanced radiation techniques, the use of better diagnostic tools nowadays can help to define the clinical target volume more precisely.

Modern dose concepts like hypofractionation (in the NeoRad trial: 40.5 Gy/2.7 Gy single dose) result in less toxicity and a smaller overall treatment time.

We are convinced that advanced techniques and modern concepts together with modern systemic treatment will improve outcomes like DFS and OS.

A major advantage of PRT in terms of a potentially better cosmetic outcome is expected for the subgroup of patients undergoing partial mastectomy or mastectomy with immediate autologous flap reconstruction. Since the flap is not irradiated, shrinkage and fibrosis of the flap are expected to be significantly lower compared to flaps receiving postoperative RT. The best evidence for this approach comes from the long-term cosmetic outcome (15 years) of 30 patients treated at the University Hospital Düsseldorf, who underwent immediate flap-based breast reconstruction after PRT and had excellent or good cosmetic results in $60 \%$ of the cases and poor results in $10 \%$ of the cases [16].

\section{PRT prior to BCS}

The theoretical advantage of PRT in case of BCS after PRT is less obvious. All of the patients in the trial will receive a tumor bed boost according to their risk profile.
Delineation of the boost volume could be more accurate in the preoperative setting than postoperatively, since there are no changes in anatomy due to the surgical procedure. Even if IORT or clip marks allow clear target definition and coverage in the postoperative setting, relatively few institutions use IORT or mark the tumor bed with clips. With regard to the latter, there is also no clear consensus on how many clips need to be set. Some may argue that the planning CT is not a perfect imaging modality for (smaller) BC either, but that also applies to postoperative RT when the tumor bed has to be defined for the boost. This typically results in smaller boost target volumes. In addition, a considerable part of the irradiated boost volume will be surgically removed in the preoperative arm. Both considerations should lead to less late fibrosis and better cosmetic results. The long-term cosmetic results after BCS were favorable in 2 phase 2 trials using PRT $(n=75)$ [17] or preoperative chemoradiation $(n=41)$ [18]. Data from randomized comparison are not available.

\section{PRT prior to Mastectomy}

Patients undergoing a skin-sparing or nipple-sparing mastectomy with immediate implant-based reconstruction have a high risk of developing capsular fibrosis and often require long-term replacement of the implant if postoperative RT is administered [19-21]. The vast majority of patients in these cohorts received conventionally fractionated RT ( 50 Gy in 25 fractions). Nowadays, hypofractionated RT (e.g., 40.5 Gy in 15 fractions) is regarded as standard of care. According to the results of large randomized trials, hypofractionated RT using $40.05 \mathrm{~Gy}$ is associated with less late effects and a better cosmetic outcome compared to conventionally fractionated RT using $50 \mathrm{~Gy}$ [22]. Thus, it is reasonable to assume that after hypofractionated RT less capsular fibrosis and a better cosmetic outcome will be observed regardless of whether preoperative or postoperative RT is used. In addition, PRT may induce less capsular fibrosis compared to postoperative RT if RT is administered at a time when the proinflammatory microenvironment at the boundary to the implant is already established [23]. This conception is indirectly supported by retrospective comparisons in some uncontrolled cohorts indicating that capsular fibrosis is less severe and requires less frequent implant replacement when PRT is administered compared to postoperative RT [20, 21].

In case of mastectomy without immediate reconstruction, the theoretical advantage of PRT is less noticeable in terms of cosmetic outcomes. However, the lower dose of the hypofractionated RT should result in less acute and late complications regardless of whether a delayed recon- 
struction is performed or not. There are studies in which hypofractionated RT was performed before mastectomy without the occurrence of relevant postoperative complications $[24,25]$. Nevertheless, according to the available data from retrospective cohorts, no significant differences in late effects between pre- and postoperative RT are expected.

\section{Toxicities after Hypofractionated PRT}

The acute side effects of RT in BC are quite moderate and they are typically limited to mild erythema and edema of the irradiated breast. In a large randomized trial $(n=923), 0.3 \%$ of the patients experienced moist skin reactions during or after hypofractionated postoperative RT with a dose of 40.05 Gy in 15 fractions, compared to $1.3 \%$ in the standard fractionation arm receiving $50 \mathrm{~Gy}$ in 25 fractions [26]. Adjuvant chemotherapy prior to adjuvant RT did not result in a significant deterioration of skin toxicity. Therefore, the acute radiation-related toxicity is expected to be moderate regardless of whether preor postoperative RT is performed.

\section{Wound Healing after PRT}

Wound healing problems, wound infections, and postoperative seroma could possibly be observed more frequently after PRT than after NACT alone. However, neither in the large cohort $(n=315)$ treated at the University Hospital Düsseldorf $[27,28]$ nor in other published cohorts using PRT or chemoradiation was an unusually high rate of complications observed [17, 18]. However, implant-based immediate reconstructions were rarely used in these cohorts. Baltodano et al. [29] evaluated the database of the American College of Surgeons National Surgical Quality Improvement Program and found 75 patients who had received PRT prior to mastectomy and immediate implant-based reconstruction versus 16,788 who had not received PRT. Morbidities at the surgical site were observed with the same frequency in both cohorts (5.3\%). In the same database, 266 patients underwent mastectomy alone after PRT and 60,773 underwent mastectomy alone without postoperative RT. Morbidities at the surgical site were reported in $4.5 \%$ after PRT and $2.7 \%$ without PRT (ns). An overview is shown in online supplementary Table 1 (see www. karger.com/doi/10.1159/000507041 for all online suppl. material). Reports on significantly higher surgical morbidities after PRT [30-35] refer almost exclusively to delayed breast reconstruction several months or even years after mastectomy and postoperative RT. Unfortunately, many authors have classified postoperative RT after mas- tectomy as PRT, which is formally correct in the context of delayed reconstructive surgery but a misnomer in our view. Accordingly, the scepticism among breast surgeons toward the use of "true" PRT is not evidence based. However, in view of the lack of randomized data, it is recommended to implement early safety checks concerning surgical morbidity if implant-based immediate reconstruction is planned in trials testing PRT.

\section{Conclusion}

In summary, there is sufficient evidence to postulate that PRT after NACT could improve DFS compared to postoperative RT, but data from a randomized trial using modern systemic treatment and radiation techniques is missing.

Overall, we are convinced that the hypothesis that PRT after NACT could lead to a significant improvement in DFS is scientifically sound. In addition, it is expected that PRT will lead to fewer late complications and better cosmetic outcomes compared to postoperative RT. Consequently, the authors of this paper plan a large clinical trial to test the before mentioned hypotheses (NeoRad trial).

\section{Statement of Ethics}

Since this is a review approval and consent are not necessary.

\section{Disclosure Statement}

The authors have no conflict of interests to declare.

\section{Founding Sources}

None.

\section{Author Contributions}

C.M. and W.B. had the idea, coordinated the work, and wrote the major part of this paper. E.B. did the literature research and contributed significantly to the text of this paper. C.M. and K.M. prepared the data for online supplementary Table 1 and Figure 1. S.C., C.S., G.F., T.K., T.F. and C.N.-K. contributed significantly to discussions on this paper and helped to design the NeoRad trial. All of the authors made substantial contributions to the conception, analysis, and interpretation of data for this work and read and approved the final version of this paper. 


\section{References}

1 Mauri D, Pavlidis N, Ioannidis JP. Neoadjuvant versus adjuvant systemic treatment in breast cancer: a meta-analysis. J Natl Cancer Inst. 2005 Feb;97(3):188-94.

2 Corradini S, Krug D, Meattini I, Matuschek C, Bölke E, Francolini G, et al. Preoperative radiotherapy: a paradigm shift in the treatment of breast cancer? A review of literature. Crit Rev Oncol Hematol. 2019 Sep;141:102-11.

3 Asselain B, Barlow W, Bartlett J, Bergh J, Bergsten-Nordström E, Bliss J, et al.; Early Breast Cancer Trialists' Collaborative Group (EBCTCG). Long-term outcomes for neoadjuvant versus adjuvant chemotherapy in early breast cancer: meta-analysis of individual patient data from ten randomised trials. Lancet Oncol. 2018 Jan;19(1):27-39.

4 Sauer R, Becker H, Hohenberger W, Rödel C, Wittekind C, Fietkau R, et al.; German Rectal Cancer Study Group. Preoperative versus postoperative chemoradiotherapy for rectal cancer. N Engl J Med. 2004 Oct;351(17): 1731-40.

5 O’Sullivan B, Davis AM, Turcotte R, Bell R, Catton C, Chabot P, et al. Preoperative versus postoperative radiotherapy in soft-tissue sarcoma of the limbs: a randomised trial. Lancet. 2002 Jun;359(9325):2235-41.

6 Thomas M, Rübe C, Hoffknecht P, Macha HN, Freitag L, Linder A, et al.; German Lung Cancer Cooperative Group. Effect of preoperative chemoradiation in addition to preoperative chemotherapy: a randomised trial in stage III non-small-cell lung cancer. Lancet Oncol. 2008 Jul;9(7):636-48.

7 Wallgren A, Arner O, Bergström J, Blomstedt B, Granberg PO, Karnström L, et al. Preoperative radiotherapy in operable breast cancer: results in the Stockholm Breast Cancer Trial. Cancer. 1978 Sep;42(3):1120-5.

8 Wallgren A, Arner O, Bergström J, Blomstedt B, Granberg PO, Räf L, et al. Radiation therapy in operable breast cancer: results from the Stockholm trial on adjuvant radiotherapy. Int J Radiat Oncol Biol Phys. 1986 Apr;12(4): 533-7.

9 Favourable and unfavourable effects on longterm survival of radiotherapy for early breast cancer: an overview of the randomised trials. Early Breast Cancer Trialists' Collaborative Group. Lancet. 2000 May;355(9217):1757-70.

10 Poleszczuk J, Luddy K, Chen L, Lee JK, Harrison LB, Czerniecki BJ, et al. Neoadjuvant radiotherapy of early-stage breast cancer and long-term disease-free survival. Breast Cancer Res. 2017 Jun;19(1):75.

11 Brackstone M, Palma D, Tuck AB, Scott L, Potvin K, Vandenberg T, et al. Concurrent Neoadjuvant Chemotherapy and Radiation Therapy in Locally Advanced Breast Cancer. Int J Radiat Oncol Biol Phys. 2017 Nov;99(4): 769-76.

12 Matuschek C, Bölke E, Roth SL, Orth K, Lang I, Bojar H, et al. Long-term outcome after neoadjuvant radiochemotherapy in locally advanced noninflammatory breast cancer and predictive factors for a pathologic complete remission : results of a multivariate analysis. Strahlenther Onkol. 2012 Sep;188(9): 777-81.
13 Huober J, Fasching PA, Hanusch C, Rezai M, Eidtmann H, Kittel K, et al. Neoadjuvant chemotherapy with paclitaxel and everolimus in breast cancer patients with non-responsive tumours to epirubicin/cyclophosphamide (EC) \pm bevacizumab - results of the randomised GeparQuinto study (GBG 44). Eur J Cancer. 2013 Jul;49(10):2284-93.

14 von Minckwitz G, Kümmel S, Vogel P, Hanusch C, Eidtmann H, Hilfrich J, et al.; German Breast Group. Intensified neoadjuvant chemotherapy in early-responding breast cancer: phase III randomized GeparTrio study. J Natl Cancer Inst. 2008 Apr;100(8):552-62.

15 Houssami N, Macaskill P, von Minckwitz G, Marinovich ML, Mamounas E. Meta-analysis of the association of breast cancer subtype and pathologic complete response to neoadjuvant chemotherapy. Eur J Cancer. 2012 Dec; 48(18):3342-54.

16 Matuschek C, Nestle-Kraemling C, Haussmann J, Bölke E, Wollandt S, Speer V, et al. Long-term cosmetic outcome after preoperative radio-/chemotherapy in locally advanced breast cancer patients. Strahlenther Onkol. 2019 Jul;195(7):615-28.

17 Calitchi E, Kirova YM, Otmezguine Y, Feuilhade F, Piedbois Y, Le Bourgeois JP. Longterm results of neoadjuvant radiation therapy for breast cancer. Int J Cancer. 2001 Aug; 96(4):253-9.

18 Bollet MA, Belin L, Reyal F, Campana F, Dendale R, Kirova YM, et al. Preoperative radiochemotherapy in early breast cancer patients: long-term results of a phase II trial. Radiother Oncol. 2012 Jan;102(1):82-8.

19 Ricci JA, Epstein S, Momoh AO, Lin SJ, Singhal D, Lee BT. A meta-analysis of implantbased breast reconstruction and timing of adjuvant radiation therapy. J Surg Res. 2017 Oct; 218:108-16.

20 Berbers J, van Baardwijk A, Houben R, Heuts E, Smidt M, Keymeulen K, et al. 'Reconstruction: before or after postmastectomy radiotherapy?' A systematic review of the literature. Eur J Cancer. 2014 Nov;50(16):2752-62.

21 Kuehlmann B, Burkhardt R, Kosaric N, Prantl L. Capsular fibrosis in aesthetic and reconstructive-cancer patients: A retrospective analysis of 319 cases. Clin Hemorheol Microcirc. 2018;70(2):191-200.

22 Haviland JS, Mannino M, Griffin C, Porta N, Sydenham M, Bliss JM, et al.; START Trialists' Group. Late normal tissue effects in the arm and shoulder following lymphatic radiotherapy: Results from the UK START (Standardisation of Breast Radiotherapy) trials. Radiother Oncol. 2018 Jan;126(1):155-62.

23 Lipa JE, Qiu W, Huang N, Alman BA, Pang CY. Pathogenesis of radiation-induced capsular contracture in tissue expander and implant breast reconstruction. Plast Reconstr Surg. 2010 Feb;125(2):437-45.

24 Thiruchelvam P, Hadjiminas D, Cleator S, Wood S, Leff D, Jallali N, et al. Neoadjuvant radiotherapy in mastectomy and immediate autologous free flap reconstruction: findings from the primary radiotherapy and DIEP flap (PRADA) pilot study. Cancer Res. 2017; 77:P3.
25 Ho AL, Tyldesley S, Macadam SA, Lennox PA. Skin-sparing mastectomy and immediate autologous breast reconstruction in locally advanced breast cancer patients: a UBC perspective. Ann Surg Oncol. 2012 Mar;19(3): 892-900.

26 Bentzen SM, Agrawal RK, Aird EG, Barrett JM, Barrett-Lee PJ, Bentzen SM, et al.; START Trialists' Group. The UK Standardisation of Breast Radiotherapy (START) Trial B of radiotherapy hypofractionation for treatment of early breast cancer: a randomised trial. Lancet. 2008 Mar;371(9618):1098-107.

27 Roth SL, Audretsch W, Bojar H, Lang I, Willers R, Budach W. Retrospective study of neoadjuvant versus adjuvant radiochemotherapy in locally advanced noninflammatory breast cancer: survival advantage in cT2 category by neoadjuvant radiochemotherapy. Strahlenther Onkol. 2010 Jun;186(6):299-306.

28 Gerlach B, Audretsch W, Gogolin F, Königshausen T, Rohn R, Schmitt G, et al. Remission rates in breast cancer treated with preoperative chemotherapy and radiotherapy. Strahlenther Onkol. 2003 May;179(5):30611.

29 Baltodano PA, Reinhardt ME, Flores JM, Abreu FM, Chattha A, Kone L, et al. Preoperative Radiotherapy Is Not Associated with Increased Post-mastectomy Short-term Morbidity: analysis of 77,902 Patients. Plast Reconstr Surg Glob Open. 2017 Mar;5(3):e1108.

30 Brooks S, Djohan R, Tendulkar R, Nutter B, Lyons J, Dietz J. Risk factors for complications of radiation therapy on tissue expander breast reconstructions. Breast J. 2012 Jan-Feb;18(1): 28-34.

31 Nahabedian MY, Tsangaris T, Momen B, Manson PN. Infectious complications following breast reconstruction with expanders and implants. Plast Reconstr Surg. 2003 Aug; 112(2):467-76.

32 Colwell AS, Damjanovic B, Zahedi B, Medford-Davis L, Hertl C, Austen WG Jr. Retrospective review of 331 consecutive immediate single-stage implant reconstructions with acellular dermal matrix: indications, complications, trends, and costs. Plast Reconstr Surg. 2011 Dec;128(6):1170-8.

33 Sbitany H, Wang F, Saeed L, Alvarado M, Ewing CA, Esserman LJ, et al. Immediate implant-based breast reconstruction following total skin-sparing mastectomy in women with a history of augmentation mammaplasty: assessing the safety profile. Plast Reconstr Surg. 2014 Jul;134(1):1-9.

34 Selber JC, Kurichi JE, Vega SJ, Sonnad SS, Serletti JM. Risk factors and complications in free TRAM flap breast reconstruction. Ann Plast Surg. 2006 May;56(5):492-7.

35 Reish RG, Lin A, Phillips NA, Winograd J, Liao EC, Cetrulo CL Jr, et al. Breast reconstruction outcomes after nipple-sparing mastectomy and radiation therapy. Plast Reconstr Surg. 2015 Apr;135(4):959-66. 